\title{
The Helium contribution from massive AGBs
}

\author{
Paolo Ventura \\ INAF - Observatory of Rome, 00040, Monte Porzio Catone (RM), Italy \\ email: ventura@oa-roma.inaf.it
}

\begin{abstract}
The helium produced by AGB and super-AGB stars is a key quantity to understand whether these objects may have been the main polluters of the interstellar medium within globular clusters, and originate a second generation of stars with a chemistry showing the imprinting of their ejecta. Helium is the most important element for this topic, as any difference in the original helium between the two populations would determine clearly distinguishable features both in the morphology of the Horizontal Branches and in the Main Sequences. We present the helium yields from massive AGB stars, and show that the results are rather robust, being approximately independent of the various uncertainties that affect the description of the evolution of these stars. The implications for the self-enrichment scenario are discussed and commented.
\end{abstract}

Keywords. Stars: abundances, AGB and post-AGB

\section{Introduction}

The traditional paradigma that stars in globular clusters (GC) are a classic example of a single stellar population, with the same age and chemistry, has been challenged in the last decades by spectroscopic evidences, showing that star-to-star differences exist in their surface chemistry, involving all the "light elements", i.e. all those species lighter than aluminium (Kraft 1994). The original idea (Denissenkov \& Weiss 2001) that these differences were due to some "in-situ" mechanism, associated to non-canonical extramixing from the bottom of the convective envelope, was disregarded by the discovery that the same patterns were also present in main sequence stars (Gratton et al. 2001), for which no active advanced nucleosynthesis could be invoked.

This opened the way to studies focused on the understanding of whether these chemical anomalies, that were surely present in the gas from which these stars formed, could be generated by the winds of intermediate-mass stars during their Asymptotic Giant Branch (AGB) phase (Ventura et al. 2001): in fact, the most massive of these objects are known to experience Hot Bottom Burning (HBB), i.e. the base of their external mantle becomes hot enough to ignite an advanced nucleosynthesis, whose products would be almost instantaneously transported to the surface, due to the rapidity of convective motions (Blöcker \& Schönberner 1991). The formation of new stellar generations in GC from the gas expelled by rapidly evolving stars belonging to the original population is commonly referred to as the "self-enrichment" scenario.

One predictions of these investigations is that massive AGB stars are helium producers, thus the gas from which the new generations of stars form is expected to be helium-rich (Ventura et al. 2001).

Based on this expectation, the stellar evolution group in Rome suggested in a series of papers that differences in the original helium content was the natural explanation of the so called "second parameter" in the interpretation of the different morphologies of the Horizontal Branches (HB) of GC, the bluest and less luminous clumps being populated by stars helium enhanced (Caloi \& D'Antona 2005, Caloi \& D'Antona 2007, D'Antona \& Caloi 2004, D'Antona \& Caloi 2008). 

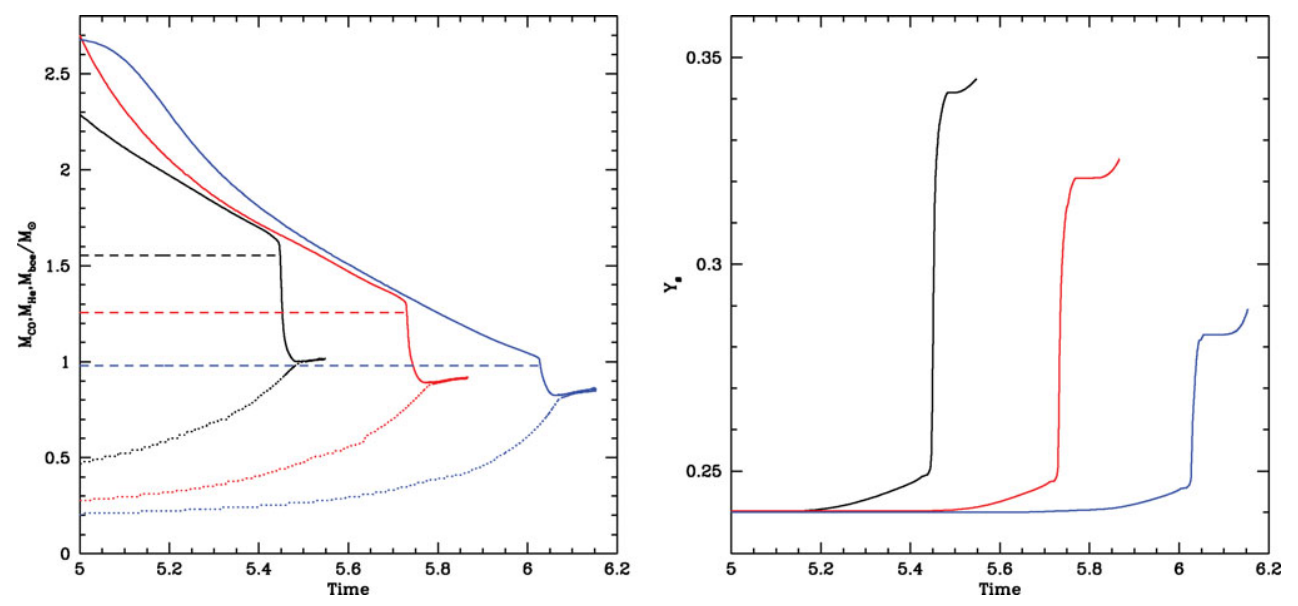

Figure 1. Left: Variation with time (in logarithmic scale, and counted from the helium exhaustion in the core) of the bottom of the convective envelope (solid), the $\mathrm{H} / \mathrm{He}$ interface (dashed), and the external border of the $\mathrm{CO}$ core (dotted) in models with initial masses 4 (right), 5 (middle), and $6 \mathrm{M}_{\odot}$ (left). Right: The helium increase following the second dredge-up for the models shown in the right panel.

This idea received a robust confirmation by the most recent photometric analysis, showing the presence of multiple main sequences in many massive GC, that can be understood only by assuming the existence of a stellar population enriched in helium (Piotto et al. 2007).

In this contribution we present the helium yields from massive AGBs, i.e. stars with mass in the range $3-6.5 M_{\odot}$, that never reach temperatures sufficiently high to burn carbon in the core. We discuss the major sources of uncertainty, and the physical processes most relevant for this particular issue. We compare these findings with the helium content of the ejecta of super-AGB stars, and find a trend monotonically increasing with the core mass.

We eventually discuss how these results may be used in the interpretation of the extendend blue tails in the HB of many GC and the presence of blue main sequences, in the context of the self-enrichment scenario for the formation and evolution of GC.

\section{The enrichment of the surface helium in intermediate-mass stars}

In stars of intermediate mass, helium burning is activated in the core in conditions of non-degeneracy; the relic of this phase is a core, made up of carbon and oxygen, evolving to conditions of progressively more enhanced degeneracy, and a helium burning buffer just above it. The ignition of this shell favours the expansion of the outer layers, with the consequent temporary extinction of the CNO burning region. During this phase the external convection sinks inwards, penetrating to layers previously touched by CNO burning, in what is commonly called "the second dredge-up" (SDU).

The left panel of Fig. 1 refers to models with masses 4,5 , and $6 M_{\odot}$; times are counted from the exhaustion of central helium. The chemistry is typical of GC with intermediate metallicity, i.e. $\mathrm{Z}=0.001, \mathrm{Y}=0.24$. The evolution of the bottom the convective envelope (solid), the border of the H-rich region (dashed), and the external border of the CO core (dotted) are shown. The $\mathrm{H} / \mathrm{He}$ interface is initially unchanged with time, because the CNO shell is not active. The base of the external mantle eventually reaches such interface, and penetrates inwards, down to the He-burning shell. This episode is accompanied by 

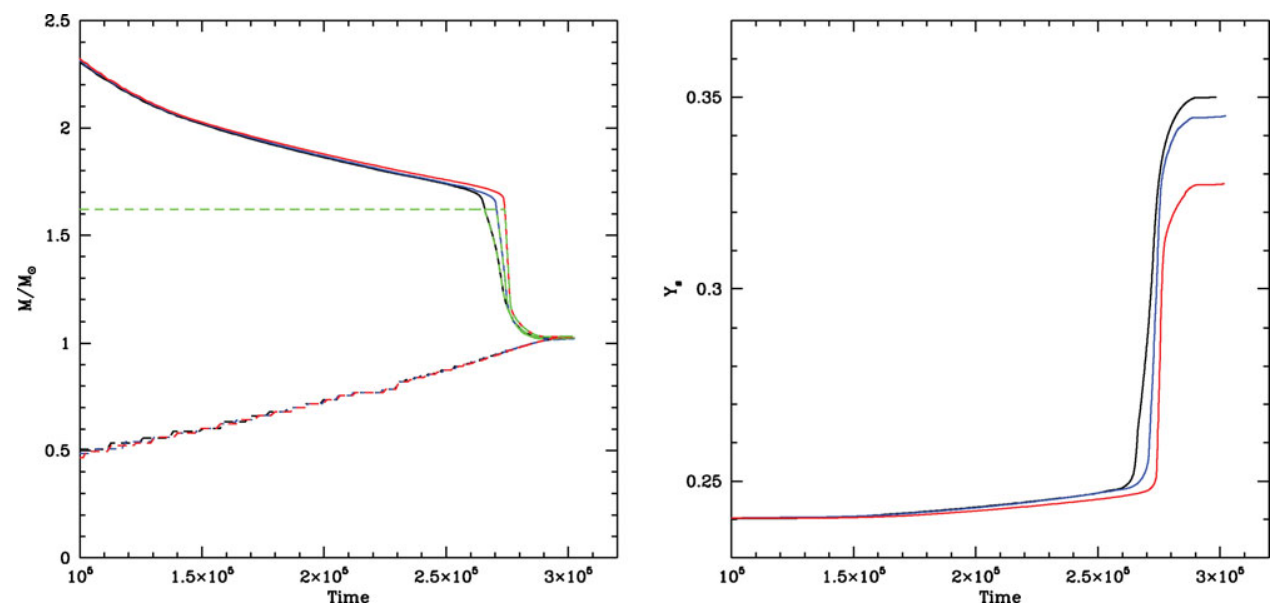

Figure 2. Left: Inwards penetration of the external envelope in a $5 \mathrm{M}_{\odot}$ models calculated with different extent of the overshooting region, i.e. with a choice of the free parameter $\zeta=0.01$ (right), 0.02 (middle), 0.05 (right). Right: The increase in the surface helium in the same models presented in the left panel.

a sudden increase in the surface helium content (see the right panel of Fig. 1), which is higher in the $6 M_{\odot}$ model, due to the largest width of the He-rich buffer.

We will see that the increase in the surface helium achieved during this phase will be crucial to determine the overall helium content of the ejecta of these stars. We therefore discuss how these results may depend on the way with which the convective/radiative interface is treated.

Modelling the AGB phase demands a diffusive approach, in which nuclear burning and mixing of chemical are coupled self-consistently (Cloutman \& Eoll 1976). The reason for this is that a non-negligible percentage of the overall nuclear energy is generated within the convective envelope, due to a partial overlapping of the external mantle with the CNO burning shell (Mazzitelli et al. 1999). Within the diffusive context, overshooting from any convective border, fixed via the Schwartzschild criterion, is modelled by an exponential decay of convective velocities within the stable region, with an e-folding distance that is commonly parametrized as $\zeta H_{p}: \zeta$ is therefore the free parameter entering the description, and providing the extent of the extra-mixing region.

The $\zeta$ adopted in this investigation is $\zeta=0.02$, in agreement with a calibration based on the observed main sequences of open clusters, given in Ventura et al. (1998). To study the sensitivity of the helium enhancement on the details of the overshooting description, we calculated three models with initial mass $5 M_{\odot}$ with $\zeta=0.01,0.02$ and 0.05 . The results of these simulations are shown in Fig. 2. We see in the left panel, showing the same quantities reported in the left panel of Fig. 1, that a greater extent of the extramixed region favours a faster inwards penetration of the convective envelope, though the innermost point reached, corresponding to the location of the He-burning shell, is approximately unchanged. The final surface helium reached at the end of this phase is thus only mildly dependent on $\zeta$, showing an increase of $\delta Y \sim 0.01$ for $\zeta$ exceeding by more than $100 \%$ the value expected on the basis of empirical calibrations.

The surface helium reached after the second dredge-up by intermediate-mass stars will be the starting point for the following phase, during which these stars experience a series of thermal pulses, provoked by the periodic ignition of a helium-rich buffer in conditions of thermal instability. Fig. 3 shows the evolution of the surface helium during the whole 
AGB phase, calculated up to the almost complete consumption of the whole external mantle. We show the mass as abscissa, to have a better feeling of the yields expected. We see in Fig. 3 that there is little space for further changes in the surface helium, that remains almost unchanged with respect to the mass fraction reached during the second dredge-up.

We conclude that the helium yield is one of the most robust results concerning the evolution of massive AGBs. Compared to the yields of other species, e.g. the CNO elements, that were shown to depend critically on the assumptions concerning the treatments of convection and mass loss (Ventura \& D'Antona 2005), the helium content of the ejecta is only modestly touched by these indeterminations, the final $\mathrm{Y}$ depending only on the abundance achieved during the second dregde-up. The conclusion from previous investigations, that suggest a maximum helium yield of $Y \sim 0.35$ expected from these stars (see, e.g., Ventura \& D'Antona 2008), is thus highly reliable.

\section{The self-enrichment scenario}

We showed that massive AGBs are expected to be efficient helium producers, providing ejecta with an average helium content up to $Y \sim 0.35$. These results confirm the speculation by Pumo et al. (2008), that the helium yields of the most massive AGBs are extremely close to the corresponding yields from the less massive super-AGBs, i.e. those stars that undergo an off-centre, degenerate, carbon ignition (Siess 2006). This fixes an increasing trend of $\mathrm{Y}$ with mass (or, more precisely, with core mass), the largest helium predicted being $Y \sim 0.40$ (see Fig. 1 in Pumo et al. 2008).

We note that this is the helium invoked by D'Antona \& Caloi (2004) to explain the presence of a detached clump of low-luminosity, blue stars, populating the HB of NGC 2808 , and it is also the same quantity required to interpret the bluest main sequence observed in the same cluster (Piotto et al. 2007).

These results open the way to new tests of the self-enrichment scenario framework. It will be possible to explain any peculiar morphology of the HBs of GC or any intrinsic width of the observed main sequences as the overlapping of an original stellar component, with the helium coming from the Big Bang, and an additional generation, born from gas that was contaminated by the winds of AGB and super-AGB stars. The degree of dilution of this gas with a pristine component is the relevant quantity to determine the helium content of the second generation, hence the features expected on the HB and the spread of the MS. The total mass and the degree of concentration of the cluster is expected to determine the extent of such a dilution process (D'Ercole et al. 2008).

\section{Conclusions}

We presented a detailed investigation of the helium yields expected from intermediatemass stars during their evolutionary history. We find that it is mainly during the second dredge-up that most of the helium enrichment is produced, with little increase in the following phase during which the stars experience a series of thermal pulses.

The helium yields show up to be scarcely sensitive to the uncertainties associated to convective overshooting, since the innermost point reached by the penetration of the convective envelope during the second dredge-up, i.e. the location of the helium-burningshell, is almost independent of the overshooting assumed. Even the impact of mass loss is negligible, because most of the mass is lost after the major phase of helium enhancement. 


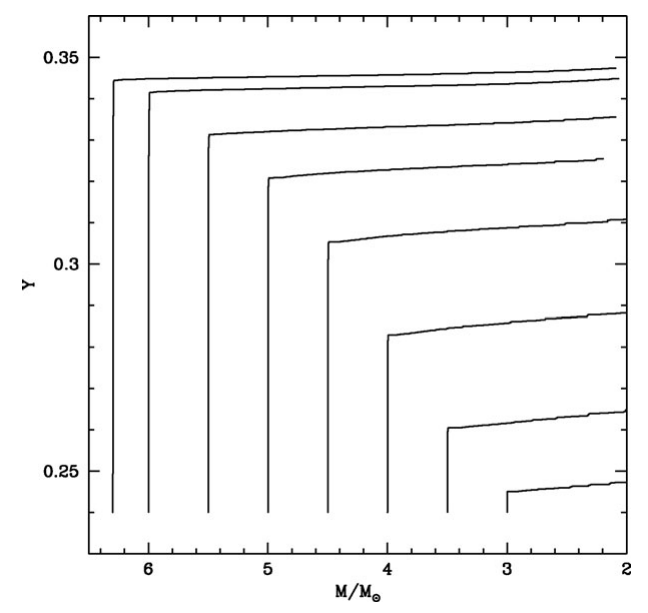

Figure 3. Evolution of the surface helium content within intermediate-mass models. Mass was chosen as abscissa, to have an idea of the chemical content of the ejecta. We note a sudden increase in the surface helium, associated to the second dredge-up, and little change in the following phase of thermal pulses.

These results indicate that helium is produced during the evolution of stars of intermediate mass, the highest abundances, $Y \sim 0.35$, being reached by the highest masses not achieving carbon-burning, i.e. $\sim 6 M_{\odot}$, this limit depending on the overshooting from the core during the H-burning phase. A higher helium enhancement is found in slightly higher mass models, the super-AGB stars, that undergo carbon ignition in a degenerate layer out of the centre.

The combination of AGB + super-AGB models produce yields with a helium content gradually increasing with mass, with a maximum of $Y \sim 0.4$. This is the value that was invoked to interpret the existence of extended blue tails in the HBs of some GC, and, more recently, the presence of a blue main sequence in NGC 2808, that is supposed to be populated by stars with a high helium content.

This result indirectly supports the self-enrichment scenario hypothesis, i.e. that in GC we observe the overlapping of more that an individual generation of stars, because new stars were born from the ashes left by the evolution of stars of intermediate mass.

\section{References}

Blöcker, T. \& Schönberner, D. 1991, A\&A (Letters), 244, 43

Caloi, V. \& D'Antona, F. 2005, A\&A, 435, 987

Caloi, V. \& D'Antona, F. 2007, $A \& A, 463,949$

Cloutman, L. \& Eoll, J. G. 1976, ApJ, 206, 548

D'Antona, F. \& Caloi, V. 2004, ApJ, 611, 871

D'Antona, F. \& Caloi, V. 2008, MNRAS, 390, 693

Denissenkov, P. \& Weiss, A. 2001, ApJ (Letters), 559, 115

D'Ercole, A., Vesperini, E., D'Antona, F., McMillan, S. L. W., \& Recchi, S. 2008, MNRAS, 391, 825

Gratton, R., Bonifacio, P., Bragaglia, A., Carretta, E., Castellani, V. et al. 2001, A\&\&A, 369, 87

Kraft, R. P. 1994, PASP, 106, 553

Mazzitelli, I., D’Antona, F., \& Ventura, P. 1999, A\&A A, 348, 846

Piotto, G., Bedin, L.R., Anderson, J., King, I. R., Cassisi, S. et al. 2007, ApJ (Letters), 661, 53 
Pumo, M. L. P., D’Antona, F., \& Ventura, P. 2008, ApJ (Letters), 672, 25

Siess, L. 2006, $A \mathscr{E} A$, 448, 717

Ventura, P., D'Antona, F., Mazzitelli, I., \& Gratton, R. 2001, ApJ (Letters), 550, 65

Ventura, P. \& D'Antona, F. 2005, A\&3A, 279, 288

Ventura, P. \& D'Antona, F. 2008, A\&3A, 479, 805

Ventura, P., Zeppieri, A., Mazzitelli, I., \& D'Antona, F. 1998, A\&A, 334, 953 